\title{
Characteristics of hybrid cells obtained by dendritic cell/tumour cell fusion in a T-47D breast cancer cell line model indicate their potential as anti-tumour vaccines
}

\author{
KARINE SERHAL ${ }^{1}$, CLAUDE BAILLOU ${ }^{2}$, NICOLAE GHINEA ${ }^{3}$, PHILIPPE FONTANGES ${ }^{4}$, \\ FRANCK P. DUPUY ${ }^{2}$, FRANÇOIS M. LEMOINE ${ }^{2}$ and ROGER LACAVE ${ }^{1}$
}

\author{
${ }^{1}$ EA3499, Université Paris 6, Laboratoire d'Histologie Biologie Tumorale, Hôpital Tenon, AP-HP; ${ }^{2}$ CNRS UMR 7087, \\ IFR113, Université Paris 6, Service de Biothérapies, Groupe Hospitalier Pitié-Salpêtrière, AP-HP; \\ ${ }^{3}$ INSERM U648, Université Paris 5; ${ }^{4}$ IFR65, Département d'Imagerie Cellulaire, \\ Université Paris 6, Paris, France
}

Received March 28, 2007; Accepted June 4, 2007

\begin{abstract}
Many strategies have been proposed to circumvent cancer development or prevent its growth. One of the promising strategies is to direct the immune response toward tumour antigens. This can be achieved by loading dendritic cells, the most potent antigen presenting cells, with tumour antigens. Fusion of dendritic cells (DC) with tumour cells is an attractive way to load the DC with all tumour antigens regardless of their immunogenicity status and the fact that they have, or not, been identified. The aim of our study was to characterise the immunophenotype of fused cells, monitor the evolution of the fusion interface and the distribution of surface antigens over time and assess for their maturation status and functionality in vitro. We used polyethylene glycol to fuse DC with Her2/neu positive breast cancer cell line T-47D. We demonstrate that false positive events accounted in flow cytometry can be identified using confocal microscopy to avoid an overestimation of fusion efficiency and to distinguish clearly hybrid cells from aggregated or phagocytosed cells. We used imaging means to demonstrate the conservation of presentation molecules (MHC II, CD1a), costimulatory molecules (CD40, CD80, CD86), as well as tumour antigens (Her2/neu, cytokeratins) in optimised conditions. Fused cells were only recognisable for $48 \mathrm{~h}$ as assessed by membrane staining and membranous antigen distribution. Fusion was necessary for their maturation to be accompanied by functional activity such as secretion of cytokines and
\end{abstract}

Correspondence to: Dr Roger Lacave, Université Pierre et Marie Curie, Laboratoire d'Histologie Biologie Tumorale, Hôpital Tenon, 4 rue de la Chine, 75970 Paris Cedex 20, France

E-mail: roger.lacave@tnn.aphp.fr

Key words: cancer, Her2/neu, immunotherapy, hybrid cell, cell fusion, dendritic cell perforin. These results suggest that hybrid cells generated by the fusion of DC and tumour cells can be easily identified and characterised using imaging techniques, and that, regarding functionality and cytokine secretion, they appear to be good candidates for anti-tumour therapies namely in breast cancer.

\section{Introduction}

Cancer is the leading cause of death worldwide accounting for 7.6 million (13\%) of all deaths. Particularly, breast cancer is the most frequent cancer among women (1). One of the most promising strategies intended to circumvent cancer growth and prevent its development, is the use of immune therapies which are intended to reinforce an existing, although weak, immune response against cancer cells. In this context, strategies have concentrated on loading tumour antigens into dendritic cells (DC), the most potent antigen presenting cells. Tumour antigens are then processed and presented to naïve $\mathrm{T}$ lymphocytes in MCH I and II context to initiate a multifactorial immune response directed against tumour antigens. Particularly DC have been loaded with antigenic peptides or proteins (2-4), DNA of defined antigens (5), whole tumour RNA (6), or loaded with tumour cell lysates (7), exosomes (8) or apoptotic cells (9). However, in order to circumvent HLA matching, antigenic mutations or uncertain immunogenicity of selected tumour associated antigens, pluriepitopic approaches for DC loading are preferred since they allow the loading of DC with identified as well as unidentified antigens. The DC/tumour cell fusion model has been proposed (10) and is widely used to load the whole tumour cell in the DC. DC provide the hybrid with an immunogenic presentation of known and unknown tumour antigens in both MHC I and II context as well as with co-stimulatory molecules which are necessary for efficient lymphocyte activation.

Hybrid DC/tumour cells have been obtained using various methods. Polyethylene glycol is the most common fusogenic agent (10-12), but other methods such as electro- 
fusion $(13,14)$ and viral fusogenic membrane glycoproteins have been used $(15,16)$. Hybrid cells have proven their efficiency in vitro with different tumour cell line models and in vivo in murine models where anti-tumour responses with a regression of pulmonary metastases have been obtained $(17,18)$. On the other hand, fused cells have proven to be safe in phases I and II clinical trials, with no toxic side effects or autoimmune reactions and with encouraging results such as clinical remissions and disease stabilisation $(19,20)$.

Whereas identification of whole fused cells has already been published $(21,22)$, most of them have relied exclusively on flow cytometric studies whereas only a few of them (23) have relied on multiple analytic methods in order to separate clearly real fused cells from aggregated and phagocytosed cells in the identification process. Moreover, little is known about the viability of fused cells in either human or murine models. Finally, little is published about the relation of the maturation status of fused cells and their functionality, especially the quantification of the cytokines secreted after $\mathrm{T}$ cell priming.

In our study, we fused human monocyte-derived DC with T-47D cells which overexpress Her2/neu, one of the most common tumour-associated antigens in breast cancer. Considering that the fusion process aims at charging the DC with unknown but potentially immunogenic tumour antigens as well as identified tumour-associated antigens, our model has the advantage of allowing easy identification of fused cells based on the expression of Her2/neu and DC markers using flow cytometry and confocal microscopy. We favour the latter because it allows a clear distinction between hybrid cells $v s$. false positives (i.e. aggregates and phagocytosed cells).

In optimised conditions for DC/T-47D fusion, our data shows that hybrid cells express membranous presentation and costimulatory molecules as well as membranous and cytoplasmic tumour antigens. However, we report discrepancies in fusion efficiency between flow cytometry and confocal microscopy assessing for false positive events in the cytometric studies. After fusion, membrane interface progressively disappears as well as DC-specific membrane staining. Whereas a simple co-incubation of DC with tumour cells in unfused control wells is sufficient to induce DC maturation, only in PEG-fused cells is maturation accompanied by efficient functional secretion of cytokines and perforin by primed T lymphocytes.

\section{Materials and methods}

Cell lines. Human breast MCF7 (HTB-22) and T-47D (HTB-133), pancreatic Capan1 (HTB-79), prostatic LNCaP (CRL-1740) cell lines were all obtained from ATCC (American Type Culture Collection, Middlesex, UK) and cultured in RPMI-1640 complete medium (RPMI $+10 \%$ foetal calf serum) (Invitrogen, Carlsbad, CA). T-47D were supplemented with $5 \mu \mathrm{g} / \mathrm{ml}$ bovine insuline.

DC culture. PBMC (peripheral blood mononuclear cells) were obtained from healthy donors with informed consent and isolated by density gradient over Lymphoprep (Abcys, Paris, France). Monocytes were separated by plastic adhesion and the monocyte-enriched fraction was cultured in 6-well plates in complete medium supplemented with $100 \mathrm{ng} / \mathrm{ml}$ recombinant human (rh)GM-CSF (granulocyte macrophage colony-stimulating factor) and $25 \mathrm{ng} / \mathrm{ml} \mathrm{rhIL-4} \mathrm{(interleukin} \mathrm{4)}$ (both from Abcys) for 6-7 days to generate immature DC. A fraction of the DC preparation underwent further maturation in the presence of $250 \mathrm{ng} / \mathrm{ml} \mathrm{CD} 40$ ligand (CD40L, Immunex, Seattle, WA) to serve as positive control only.

Cell characterisation. Tumour cells were characterised using unconjugated mAB c-neu (Oncogene, EMD Biosciences, Darmstadt, Germany), PAN-cytokeratine (Novocastra, Norwell, MA). They were shown to express MHC I, but they expressed neither MCH II nor co-stimulatory molecules which were characteristics of DC. The secondary antibody was PE- (phyco-erythrin) or -FITC (fluorescein isothiocyanate) conjugated $\mathrm{F}(\mathrm{ab}) 2$ goat anti-mouse (Immunotech, Marseille, France). For intracellular staining of tumour cells, either IntraPrep kit from Immunotech was used or a protocol of 15-min fixation in paraformaldehyde (PFA) $2 \%$ followed by a 2-min permeabilisation with Triton X-100 (0.15\%) was employed. To immunophenotype DC, monoclonal antibodies (mABs) to the following antigens were used: HLADR-PE (Beckman Coulter, Fullerton, CA, USA), CD1a-PE, CD86-PE, CD40-PE, HLA ABC-FITC, CD80-FITC were obtained from Immunotech. Isotype control mABs included IgG1a-PE, IgG2a-PE and IgG1a-FITC (Immunotech). At least 100,000 gated events were analysed using an EPICS Elite $^{\mathrm{TM}}$ and Expo32 software (Beckman Coulter). In some experiments, double positive fraction was sorted using a FACS Star Plus flow cytometer (Becton-Dickinson, San Jose, CA).

Cell staining. Tumour cell lines were dyed green with 0.1-0.15 $\mu \mathrm{M}$ of CMFDA (5-chloromethylfluorescein diacetate) according to the manufacturer's protocol (Molecular Probes, Invitrogen) and DC were dyed red with $1 \mu \mathrm{M}$ of CMTMR [(5-(and-6)-(((4-chloromethyl)benzoyl)amino) tetrame thylrhodamine) - mixed isomers]. Briefly, cells were incubated for $30 \mathrm{~min}$ with dyes diluted in complete medium, washed and then incubated for $30 \mathrm{~min}$ in complete medium.

Cell fusion. After staining, DC and tumour cells (TC) were mixed before fusion at different DC:TC ratios $(1: 1$; $3: 1 ; 10: 1)$. A total and fixed cell number of $10^{7}$ cells was fused using 50\% polyethylene glycol (PEG) 1500 (Roche Diagnostics, Basel, Switzerland) according to manufacturer's instructions. Briefly, cells were spun by centrifugation and supernatant was removed. The pellet was broken by a $2-\mathrm{min}$ pipetting in $1 \mathrm{ml}$ of prewarmed PEG $1500\left(37^{\circ} \mathrm{C}\right)$. Then, $1 \mathrm{ml}$ of prewarmed RPMI was slowly added over $1 \mathrm{~min}$ and then $3 \mathrm{ml}$ of RPMI were added over a period of $3 \mathrm{~min}$. The mixture was then incubated for $5 \mathrm{~min}$ at $37^{\circ} \mathrm{C}$ in a $15 \mathrm{ml}$ final volume of RPMI, then centrifuged for $5 \mathrm{~min}$ at $1500 \mathrm{rpm}$. Fusion products were suspended in $10 \mathrm{ml}$ complete medium unless otherwise specified and plated in two $60 \mathrm{~mm}$ Petri dishes overnight. As a control for spontaneous double fluorescent events, non PEG-treated DC and TC were cocultured in the same 1:1; 3:1 and 10:1 DC:TC ratio. Unless otherwise stated, cells were harvested $18 \mathrm{~h}$ after fusion. 
Table I. Range and means of double positive events obtained by fusing different tumour cell types with DC.

\begin{tabular}{lcccc}
\hline & T-47D & LNCaP & Capan-1 & MCF7 \\
\hline Mean percentages of double positive (events \pm SEM) & $17.1 \pm 3$ & $19 \pm 4.5$ & $13.1 \pm 3.2$ & $12.1 \pm 2.3$ \\
Range of double positive events (\%) & $7.7-29$ & $11.7-27.3$ & $7.1-25.6$ & $7.1-18$ \\
Number & 7 & 3 & 5 & 4 \\
\hline
\end{tabular}

Regardless of the type of tumour cell fused with DC, no statistical difference is observed using Kruskal-Wallis test.

Supernatant was gently separated from the adherent fraction, cells were incubated in Trypsine-EDTA (Invitrogen) to eliminate aggregates and viability was assessed using trypan blue.

Characterisation of fusion products. Fusion products were analysed using flow cytometry and confocal microscopy. Tumour cells were stained with the FITC dye prior to fusion. Cells were then fused and incubated overnight. Cells were harvested, stained with HLA-DR, CD1a, CD40, CD86-PE conjugated mABs, fixed in $2 \%$ PFA and analysed by flow cytometry or cytospun and observed using a Leica confocal microscope (Wetzlar, Germany). To look for the expression of tumour antigens (cytokeratins and Her2/neu) in hybrid cells, DC were stained with CMFDA or CMTMR prior to fusion and then fused with T-47D. Fusion products were incubated with an anti-PAN-cytokeratin (PE) or an antiHer2/neu antibody (FITC). Fused cells were also studied for dual HLA-DR-Her2/neu expression. To do so, cells were fused and $18 \mathrm{~h}$ later, harvested, fixed in 2\% PFA, cytospun and incubated with monoclonal HLA-DR and polyclonal anti-Her2/neu primary antibodies. Goat anti-mouse IgG Alexa 546 and goat anti-rabbit IgG Alexa 488 (Invitrogen) were used as secondary antibodies. Nuclei were stained with Toto-3 (Invitrogen) and cells were examined under a confocal microscope. Representative cell samples were viewed in the $(\mathrm{x}, \mathrm{y})$ planes at indicated time points and the images recorded.

Mixed lymphocyte reaction. DC, T-47D, the control unfused mix of DC + T-47D and fused cells DC/T-47D (stimulator cells) were exposed to $50 \mathrm{~Gy}$ of ionising radiation and then incubated at different ratios with $10^{5}$ allogeneic $\mathrm{CD}^{+} \mathrm{T}$ lymphocytes (responder cells) in 96-well round bottom culture plates. $\mathrm{CD}^{+} \mathrm{T}$ lymphocytes were purified using antiCD3 magnetic beads (MiltenyiBiotec, Bergisch Gladbach, Germany). The plates were incubated 6 days in a humidified atmosphere under $5 \% \mathrm{CO}_{2}$ at $37^{\circ} \mathrm{C}$. Cells were co-incubated at responder:stimulator $(\mathrm{R}: \mathrm{S})$ ratios of $10: 1$ and $20: 1$. The proliferation of lymphocytes was determined by the uptake of $2 \mu \mathrm{Ci}\left[{ }^{3} \mathrm{H}\right]$-thymidine (Amersham, Saclay, France) for the last $16 \mathrm{~h}$ of incubation. Allogeneic $\mathrm{CD} 3^{+} \mathrm{T}$ lymphocytes incubated with $5 \mu \mathrm{g} / \mathrm{ml}$ OKT3 and $600 \mathrm{UI} / \mathrm{ml} \mathrm{IL-2} \mathrm{were}$ considered as positive control. Results are the mean \pm SEM of triplicate wells on at least 3 different experiments.

Assessment of cytokine and perforin secretion. To determine the secretion of cytokines by cells, irradiated DC, T-47D, a mix of unfused DC + T-47D and fused DC/T-47D, were cultured with autologous purified $\mathrm{CD}^{+} \mathrm{T}$ lymphocytes. Cells $\left(10^{5}\right)$ were seeded in 24 -well plates with $10^{6}$ autologous $\mathrm{T}$ cells in $1 \mathrm{ml}$ of complete medium. After 3 days of culture, half of the medium was changed and IL-2 was added to a final concentration of $20 \mathrm{UI} / \mathrm{ml}$. Half of the medium was changed every 3 days. Supernatants were collected at days 3, 6 and 9 and IFN $\gamma$ and IL-10 levels were determined by ELISA (R\&D Systems, Minneapolis, MN). Perforin levels were determined by ELISA using Diaclone Eli-Pairs (Diaclone, Besançon, France). Results shown consider secretion of cytokines induced by DC as basal levels and are the mean \pm SEM of triplicates on at least 4 different experiments.

Statistical analyses. Statistics were done using Kruskal-Wallis and Student's t-test on StatView ${ }^{\circledR}$ software. Differences were considered statistically significant for $\mathrm{p} \leq 0.05$.

\section{Results}

Percentage of double fluorescent events is independent of tumour cell type. To determine if fusion efficiency was dependent on the tumour cell line used, 4 tumour cell types were stained with CMFDA and DC were stained with CMTMR prior to fusion. Double positive events were considered representative of fusion efficiency. Regardless of the tumour cell type fused with DC, mean double positive events ranged between 12 and 19\%. Mean percentage of double positive events was $12.1 \%$ for MCF7 (range 7.1-18\%), $13.1 \%$ for Capan-1 (range 7.1-25.6\%), 17.1\% for T-47D (range $7.7-29 \%$ ) and $19 \%$ for $\mathrm{LNCaP}$ (range 11.7-27.3\%) (Table I). Mean percentage of spontaneous double positive events in control wells was never greater than $3 \%$ for all cell types. These results do not show statistical differences in double positive events yielded. With a fusion efficiency of approximately $17 \%$, our results are in agreement with others (11) and thus validate our fusion model. Therefore, $\mathrm{T}-47 \mathrm{D}$ mammary cell line was used in all other experiments.

Optimised conditions are DC:TC ratio of 3:1 and fused cells are mostly non-adherent. Cells were fused at different DC:TC ratios to determine optimised conditions for the production of fused cells. Balance between productivity and cell viability $18 \mathrm{~h}$ after fusion was the criteria for choosing the best conditions for fusion. A total cell number of $10^{7}$ cells was fused irrespective of DC:TC ratio. As shown in Table II and Fig. 1a, production of fused cells increased with DC:T-47D ratio. On the contrary, cell viability decreased 
Table II. DC:T-47D ratio of 3:1 produces higher percentage of fused cells with higher viability.

\begin{tabular}{lccc}
\hline $\begin{array}{l}\text { DC:T-47D } \\
\text { ratio }\end{array}$ & $\begin{array}{c}\text { Mean \% } \\
\text { of hybrid } \\
\text { cells }\end{array}$ & $\begin{array}{c}\text { Viability }(\%) \\
5 \text { min } \\
\text { after fusion }\end{array}$ & $\begin{array}{c}\text { Viability (\%) } \\
18 \mathrm{~h} \\
\text { after fusion }\end{array}$ \\
\hline $1: 1$ & 13.1 & 56.9 & 14.5 \\
$3: 1$ & 17.1 & 55.7 & 32.4 \\
$10: 1$ & 19.2 & 41.9 & 3.1 \\
\hline
\end{tabular}

$n \geq 3$ experiments

with DC:T-47D ratio when cells were harvested $18 \mathrm{~h}$ after fusion. Since mortality of PEG-incubated T-47D alone was around $11 \%$ (data not shown), low viability observed for higher DC:TC ratio can be attributed to PEG toxicity vis-àvis DC. 3:1 ratio was therefore considered the best in our model, with a $17 \%$ mean production of double positive events and $55.7 \%$ viability. DC:T-47D ratio of 3:1 was used in every following experiment.

When adherent and non-adherent fractions were analysed separately for double positive events $18 \mathrm{~h}$ after fusion using flow cytometry, the non-adherent (NAdh) fraction showed almost 3 times more double positive events than adherent (Adh) fraction (Fig. 1b). Because non-adherent fraction is more concentrated in fused cells, further experiments were conducted on this fraction and the adherent fraction was discarded. On the other hand, unfused control cells did not show any double stained cells.

Flow cytometry overestimates the production of hybrid cells. Discrepancies between methods used to analyse hybrid cell production were shown when comparing flow cytometry and confocal microscopy. Indeed, when observed with a confocal microscope, real double positive fused cells show a yellow cytoplasm assessing for the blending of the two cytoplasmic markers (Fig. 2a, i). They can be clearly distinguished from double fluorescent false positives non-fused cells (Fig. 2a, ii-iv). They may include a large amount of aggregates and phagocytosed fragments of dead cells from the partner cells. These latter cells cannot be distinguished using flow cytometry. When fluorescence is monitored in one single yellow cell using confocal microscope, 2 overlaid curves (channels 1 and 2 for CMFDA and CMTMR, respectively) are representative of the blending of the 2 cytoplasmic markers (Fig. 2b). Therefore, we conclude that percentages of double positive events obtained using flow cytometry clearly overestimate real percentages of fused cells. When sorted and stained with May Grunwald Giemsa, real hybrid multinucleated cells are recognisable, in enriched fraction, to histological different nuclei as shown by nucleoli and chromatin structures (Fig. 2c, iii-v). DC have a small nuclei, sometimes invisible nucleoli and homogeneous chromatin (Fig. 2c, i), tumour cells have irregular nuclei and voluminous purple stained nucleoli representative of a high synthesis activity and very dense chromatin (Fig. 2c, ii). a)

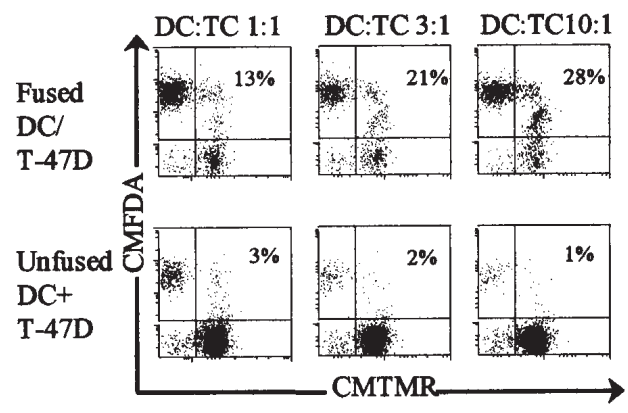

b)

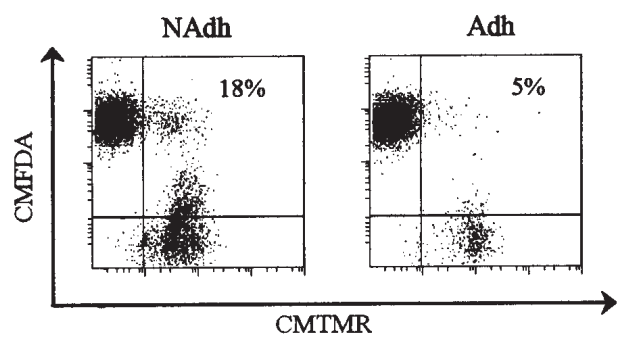

Figure 1. Optimised ration for DC/T-47D production is DC:TC $=3: 1$ and the non-adherent fraction is enriched in fused cells. (a) Tumour cells (CMFDA) are fused to DC (CMTMR). Double positive events appear clearly in the upper panel and production increases with DC:TC ratio. Double positive events are negligible in control unfused wells (lower panel). (b) Ratio of non-adherent (NAdh) to adherent (Adh) cells is about 3. Here we show one representative experiment out of five with $\mathrm{Nadh} / \mathrm{Adh}$ ratio (range 1.4-4.8) representative of the higher concentration of fused cells in the non-adherent fraction.

Presentation molecules and co-stimulatory molecules are expressed on hybrid cells. Expression of presentation molecules, such as MHC II and CD1a, and co-stimulatory molecules such as CD40, CD86 and CD80, can be seen on the membrane of hybrid cells using confocal microscopy (Fig. 3). For these experiments, tumour cells were stained with a cytoplasmic marker (either CMFDA or CMTMR) prior to fusion, and then, after fusion with DC, fused cells were incubated with conjugated antibodies directed against DC. Hybrid cells appear double stained with both cytoplasmic and membranous markers. Conservation of presentation molecules and co-stimulatory molecules on hybrids indicates that they are likely to be functional and capable of activating T lymphocytes. Similarly, hybrid cells express tumour antigens, either membranous Her2/neu or cytoplasmic cytokeratins as demonstrated in Fig. 3. The arguments brought forward by confocal microscopy as opposed to flow cytometry are the clear identification of isolated cells as opposed to aggregates or phagocytosed debris. These findings were supported by flow cytometry (data not shown).

Hybrid cells evolve over time as fusion membrane progressively disappears and Her2/neu spreads. In order to study the phenotypic evolution of fused cells over time, particularly the evolution of the fusion interface, hybrid cells were double stained with HLA DR-PE and Her2/neu-FITC after fusion. To exclude aggregated phenomena, harvested cells were systematically incubated in trypsin-EDTA for $10 \mathrm{~min}$. Staining of the membranes allows easy identification of fused cells, and hybrid cells appear as early as $4 \mathrm{~h}$ after fusion (Fig. 4). At $\mathrm{t}=4 \mathrm{~h}$, staining in the fusion interface is weak 
b)

a)

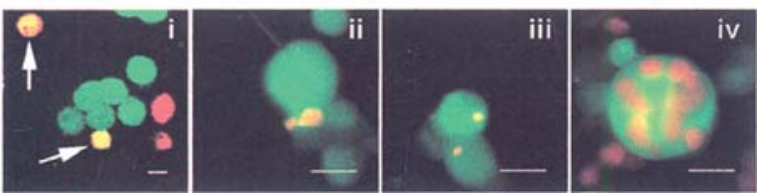

c)

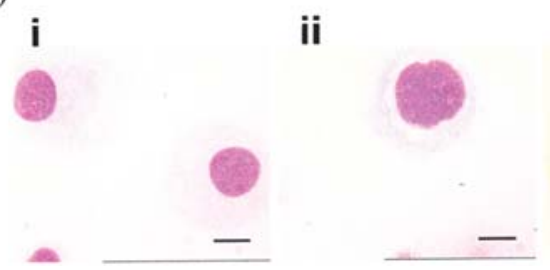

iii

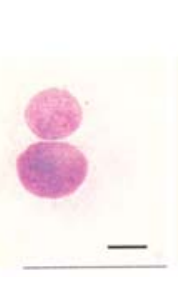

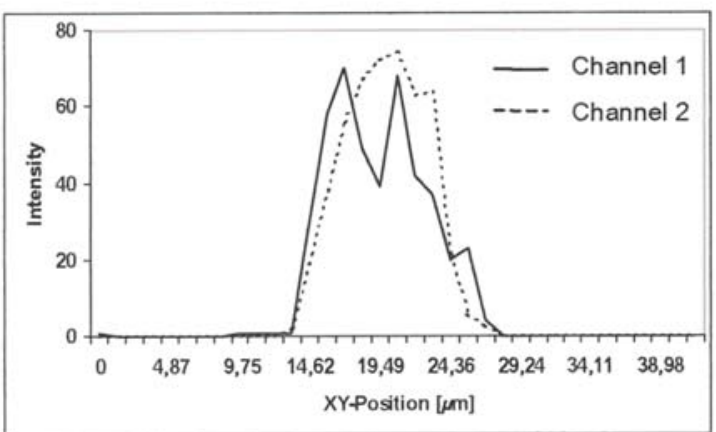

iv

$\mathbf{V}$

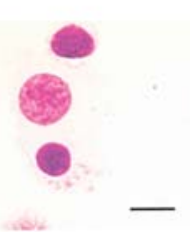

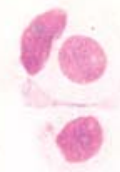

Figure 2. Confocal microscopy allows easy identification of false positive events. (a) Confocal microscopy shows green and red unfused T-47D and DC and fused cells appear as yellow double stained cells containing both cytoplasmic markers (i, arrows). They can be easily distinguished from double fluorescent false positives such as phagocytosed cells or cell debris (ii,-iv). Scale bar, $10 \mu \mathrm{m}$. (b) A double peak representing both CMFDA (-) and CMTMR (- - -) fluorescence in one fused cell can be measured using confocal microscopy assessing for cytoplasm blending. (c) Sorted double positive elements from nonadherent fraction observed after May-Grunwald Giemsa Staining appear multinucleated with histological different nuclei [DC in (i), T-47D in (ii)]. Hybrid cells observed are bi or multi-nucleated and vary in size and shape (iii-v). Scale bar, $20 \mu \mathrm{m}$.

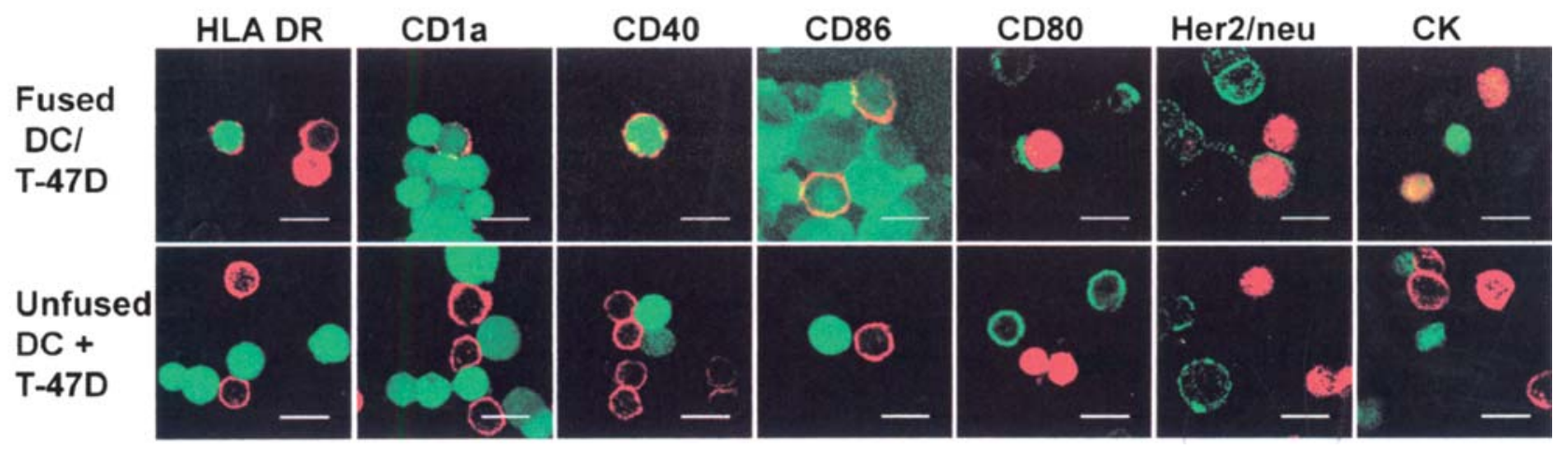

Figure 3. Hybrid cells express presentation, co-stimulatory molecules and tumour antigens. Fused cells appear double fluorescent with a double stained cytoplasm and membrane. For each, presentation, co-stimulatory and tumour antigen appear as a membranous fluorescence, except for cytokeratins which are cytoplasmic. No double staining is apparent in the control unfused cells presented in the lower panel. Staining: For HLA DR, CD1a, CD40 and CD86, tumour cells are stained with CMFDA-FITC and surface molecules with -PE. For CD80 and CK, TC are stained with -PE dyes and CD80 and CK stained with an FITC dye. For Her2/neu, DC are stained with cytoplasmic CMTMR and Her2/neu with FITC. Scale bar, $20 \mu \mathrm{m}$.
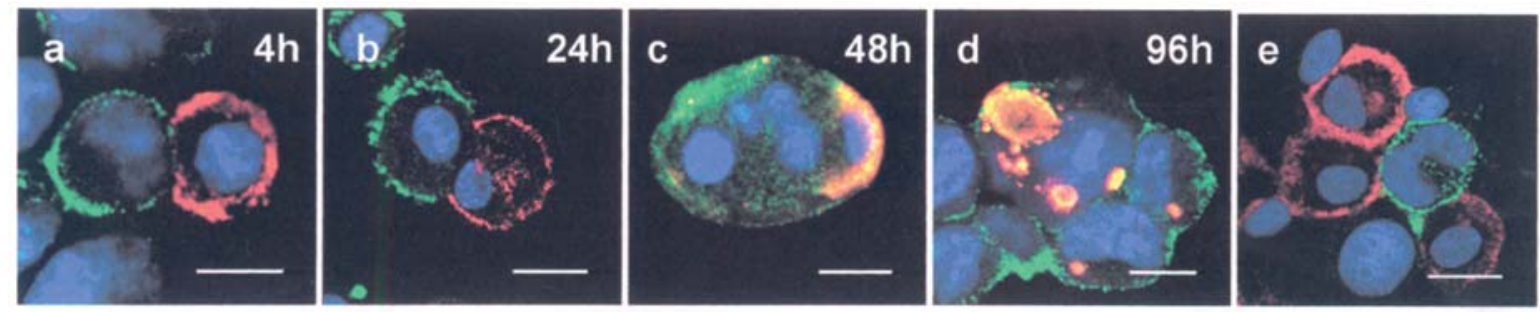

Figure 4. Evolution of the fusion membrane and surface antigen distribution over time. Four hours after fusion (a), staining of the membrane at the fusion interface with HLA DR (PE) and Her2/neu (FITC) is light but still apparent. The membrane interface vanishes over time (b) to become completely blended after $48 \mathrm{~h} \mathrm{(c)} \mathrm{with} \mathrm{a} \mathrm{slight} \mathrm{but} \mathrm{visible} \mathrm{distribution} \mathrm{of} \mathrm{the} \mathrm{tumour} \mathrm{antigen} \mathrm{Her2/neu} \mathrm{all} \mathrm{around.} \mathrm{Ninety-six} \mathrm{hours} \mathrm{after} \mathrm{fusion} \mathrm{(d),} \mathrm{fused} \mathrm{cells} \mathrm{are} \mathrm{no} \mathrm{longer}$ identified by membranous staining; only Her2/neu is homogeneously distributed. Staining of the membranes stays very clear in control unfused cells (e) as opposed to fused cells (here at $\mathrm{t}=24 \mathrm{~h}$ representative of all time points). Scale bar, $20 \mu \mathrm{m}$. 
a)

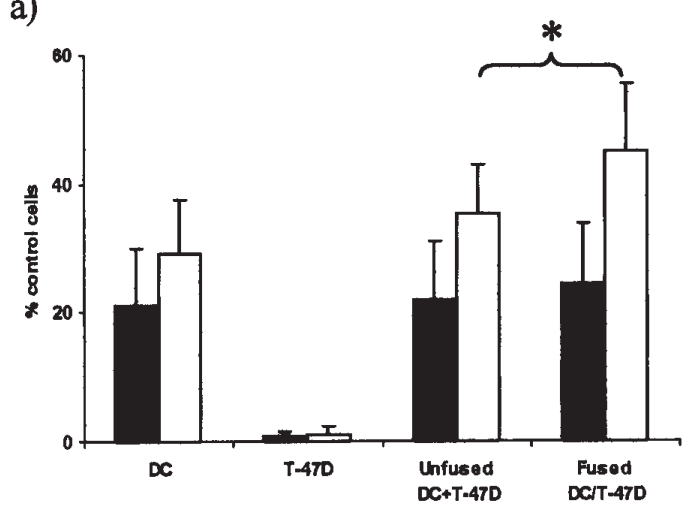

b)

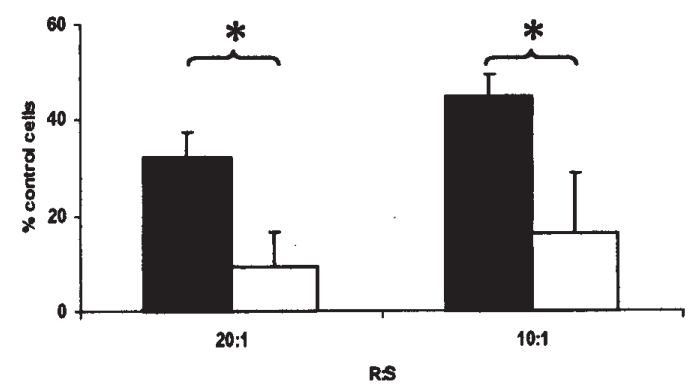

Figure 5. Fused cells are efficient in activating allogeneic T lymphocytes. (a) Fused cells induce the proliferation of allogeneic $\mathrm{CD}^{+} \mathrm{T}$ cells especially at the $\mathrm{R}: \mathrm{S}=10: 1$ ratio (white bars). Results are expressed as percentage of positive control (allogeneic $\mathrm{CD}^{+} \mathrm{T}$ cells + OKT3 + IL-2). R:S=20:1 for black bars and 10:1 for white bars. (b) Non-adherent fraction (black bars) is more potent in inducing the activation of $\mathrm{CD}^{+} \mathrm{T}$ cells than adherent fraction (white bars). $n \geq 3$ experiments.

though still apparent (Fig. 4a). Twenty-four hours after fusion, staining is restricted to membranes and does not appear anymore in the fusion interface (Fig. 4b). Finally, $48 \mathrm{~h}$ after fusion (Fig. 4c), the fusion membrane seems to have totally disappeared and the cells are blending into one another. HLA DR molecule at this time is still heterogeneously distributed whereas Her2/neu is distributed all around the membrane. Ninety hours after fusion (Fig. 4d), regardless of the presence of GM-CSF and IL-4 in the medium, we could not find membranous staining of HLA DR and, whereas Her2/neu staining was still present, HLA DR appeared as intracellular isolated staining. The change in HLA DR distribution is inherent to fused cells, since DC and control unfused DC co-incubated with T-47D show normal HLA DR distribution for $>96 \mathrm{~h}$. Control non-fused cells, as opposed to PEG-fused cells, keep distinct membrane staining throughout all time points, even if physically close (Fig. 4e). In conclusion, clear membranous distribution of HLA DR molecules in PEG fused cells, in our model, does not exceed $48 \mathrm{~h}$. After this time, only Her2/neu staining is observable. During the fusion, and later in the evolutionary process of hybrid cells, we have no proof of nuclei fusion.

Functionality is not altered in non-adherent fused cells. As shown in allogeneic MLR, fused cells maintain the capacity of antigen presentation and activation of allogeneic T lymphocytes (Fig. 5a). The activation induced by total fused cells

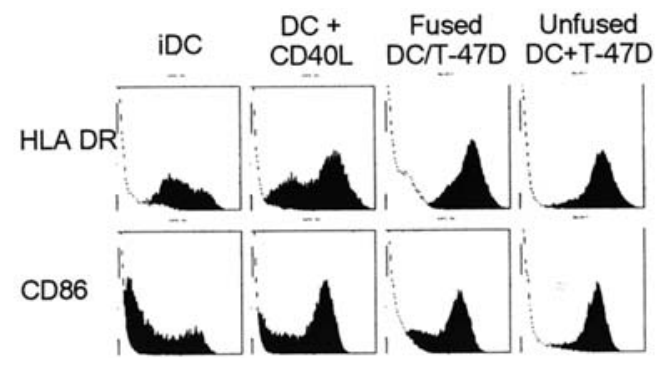

Figure 6. Fused cells show a mature phenotype. PEG fusion as well as simple co-incubation with tumour cells is sufficient for DC to acquire a mature phenotype when compared to CD40L-matured DC.

a)

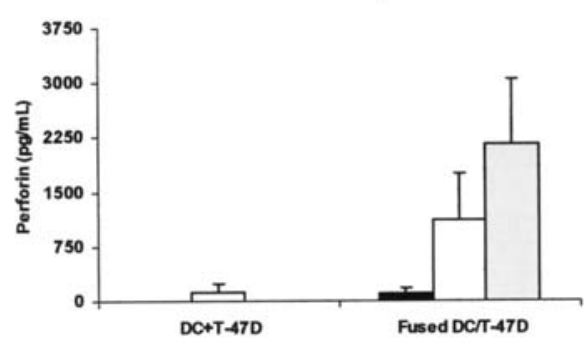

b)
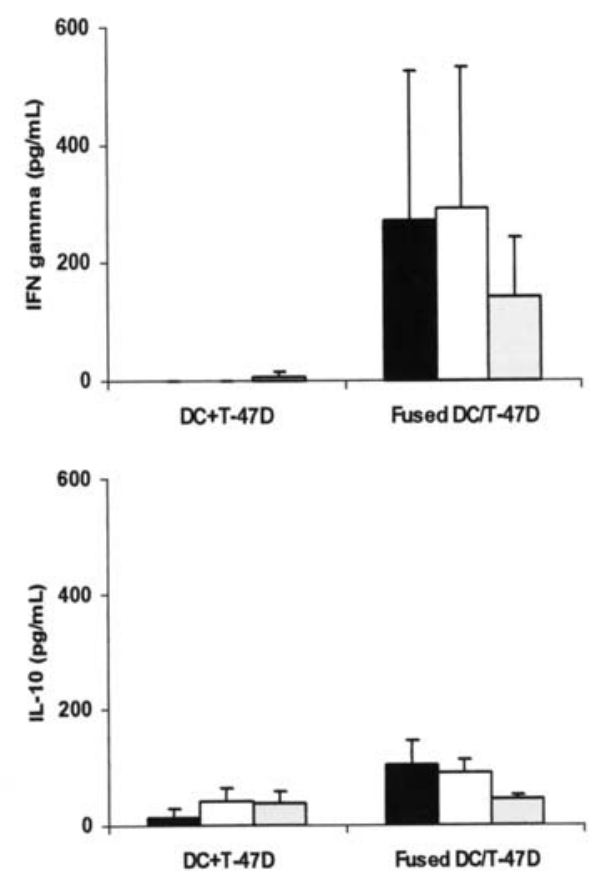

Figure 7. Fused cells induce the secretion of perforin and Th1 cytokines. (a) Fused cells induce higher levels of perforin by primed $\mathrm{T}$ cells than control unfused cells. (b) Levels of IFN $\gamma$ secreted over time (upper panel) peak at day 6 and levels of IL-10 induced are low (lower panel). The secretion of both these cytokines is higher than the secretion induced by control unfused cells. Results are the means of 4 experiments \pm SEM compared to basal levels induced by DC. Black bars, day 3; white bars, day 6; gray bars, day 9 .

is slightly higher than that induced by the unfused control cells, and results show a slightly better activation in the $\mathrm{R}: \mathrm{S}=10: 1$ ratio $(\mathrm{p}=0.015)$ for fused cells. The close activation rates of fused vs. unfused cells is attributable to the allogeneic 
reaction of DC in both preparations. However, this does not preclude their functionality. When differential activation of $\mathrm{T}$ lymphocytes by non-adherent and adherent fractions is investigated, we show that non-adherent fraction, which is enriched in fused cells, is, for all ratios investigated, significantly more potent in activating T lymphocytes (Fig. 5b) ( $\mathrm{p}=0.0003$ for $\mathrm{R}: \mathrm{S}=20: 1$ and $\mathrm{p}=0.001$ for $\mathrm{R}: \mathrm{S}=10: 1)$. Therefore, we conclude that fused cells are functional and capable of initiating $\mathrm{T}$ cell proliferation in vitro confirming previously published results.

Only in fused cells is maturation accompanied by functional activity. Cell surface expression of MHC II and CD86, commonly used as maturation markers, was shown to be upregulated on fused cells as well as on co-cultured unfused cells compared to immature DC (iDC) as shown in Fig. 6. For fused cells, mean fluorescence intensity increased from 25.7 to 68.2 for HLA-DR and from 19.6 to 24 for CD86 reaching values close to $\mathrm{CD} 40 \mathrm{~L}-$ matured $\mathrm{DC}$ which served as positive control (respectively 46 and 22.8). This upregulation observed with both fused and unfused cells and compared to CD40L-matured DC, suggests that simple co-incubation of DC with T-47D, as well as PEG fusion of the 2 cell types, is sufficient to induce maturation. This phenotypic maturation is however not necessarily correlated to functionality. Indeed, as demonstrated for the first time in a human model, perforin is induced by fused cells at concentrations significantly higher than the ones induced by control unfused cells at days 6 and 9 ( $\mathrm{p}=0.016$ and $\mathrm{p}=0.001$ respectively) (Fig. 7a). This secretion of perforin suggests an activation of $\mathrm{CD}^{+}$cytotoxic $\mathrm{T}$ lymphocytes. Like perforin, levels of IFN $\gamma$ induced by DC/T-47D fused cells are significantly higher than levels induced by control nonfused cells ( $\mathrm{p}=0.042$ and $\mathrm{p}=0.010$ at days 6 and 9 respectively). Fig. 7b (upper panel) shows that this IFN $\gamma$ secretion is high starting at day 3 and peaks at day 6 . Secretion of the IL-10 (Fig.7b, lower panel) is induced at lower levels and decreases over time. Fused cells induce a significantly higher secretion of IL-10 than unfused control cells only at days 3 and $6(\mathrm{p}=0.018$ and $\mathrm{p}=0.017$ respectively). The differential secretion of cytokines and perforin induced by fused cells shows that fused cells are fully functional and capable of activating naïve $\mathrm{T}$ cells. Fused cells are hence potentially more efficient candidates for anti-tumour therapies than cocultivated cells.

\section{Discussion}

The combination of established conventional tumour therapies with immunotherapy is to be investigated intensely in the coming years. Because of their low toxicity, DC-based vaccines have emerged as a promising tool in cancer immunotherapy. For this, priming of naïve T cells and the triggering of an anti-tumour immune response are necessary and, for efficient immune response, these vaccines face challenges such as choosing the right antigen and generating the right type of immune response. The fusion of DC with allogeneic tumour cells is an effective approach to introduce both identified and unidentified tumour associated antigens into DC. Loaded DC would then have to process tumour antigens and present them to naïve $\mathrm{T}$ cells in order to induce a cytotoxic anti-tumour immune response. The response to DC fused with allogeneic cells has been shown to induce a high proliferation of $\mathrm{T}$ cells and comparable cytotoxicity to the DC fused with autologous tumour cells (24). Therefore, and because tumour cell lines are more easily available compared to viable breast cancer cells from primary lesions, more stable and more easily cultivated in vitro, we validated our model by fusing DC with the Her2/neu bearing tumour cell line T-47D. The choice of this model was supported by the overexpression of Her2/neu in approximately $25 \%$ of breast cancer cases as well as in numerous additional human adenocarcinomas (25).

In our study, we relied on imaging means provided by confocal microscopy to study fusion efficiency and immunophenotype of hybrid cells. Indeed, even though our findings were supported by flow cytometry and in that, met previously published results $(21,22)$, we observed discrepancies, particularly in fusion yields and efficiency, between the methods. We favoured confocal microscopy for the study of hybrid production and surface molecule conservation because it allowed clear distinction of isolated fused cells as opposed to aggregated cell or phagocytosed cells/debris which could account for double positive events using flow cytometry.

Non-adherent fused cells demonstrated good functionality of presentation and co-stimulatory molecules by activating allogeneic T lymphocytes and these finding are in agreement with previous reports on both breast (26) and colorectal cancer (17). We concentrated target cells by using the nonadherent enriched fraction after fusion to minimise the activation that could be, in part, attributable to apoptotic/ necrotic cells generated by PEG toxicity. On the contrary, tumour cells do not induce $\mathrm{T}$ cell proliferation partly because they lack co-stimulatory molecules (27) and are unable to process tumour antigens (28). Immunophenotypic characterisation of the fused cells is done by concentrating target cells in the non-adherent fraction and fused cells are easily recognisable after May-Grunwald Giemsa or specific membranous staining. Our study is the first to point out an evolution of the fusion process which presents morphological similarities with the model developed by Riske et al for the fusion of lipid layers (29). Indeed, we show the progressive disappearing of the membrane at the fusion interface in hybrid cells and, simultaneously, an evolution of surface antigen distribution. Hybrid cells, as assessed by membranous distribution of antigens, are no longer present after $48 \mathrm{~h}$. Instead, we observed large multinucleated group of cells expressing surface tumour antigen but only intracellular localised DC-specific staining. The progressive disappearing of HLA DR staining after $48 \mathrm{~h}$ could be due, on the one hand, to the phagocytosis of dead DC debris by tumour cells. This could explain the relatively short presence of hybrid cells (48 h). But, on the other hand, the fusion products could lose the DC phenotype and be overtaken by tumour phenotype. Further experiments are however needed to confirm these hypotheses.

Our data also clearly indicate that maturation of DC is not necessarily linked to functionality. Co-culture with tumour cells, with no PEG exposition, was sufficient to 
induce maturation of DC in these wells. However, even when mature, the DC in unfused wells are not fully functional. Indeed, cytokine secretion induced by subsequent activation of $\mathrm{T}$ cells by unfused cells stays below basal levels induced by DC. On the contrary, fused cells induce the secretion of high levels of IFN $\gamma$ and perforin. Both these secreted products are representative of an immune response directed toward a cytotoxic path. Other authors have reported high percentages of cytokine-secreting $\mathrm{T}$ cells using intracellular staining (24) or their secretion in different fusion models (30). To these very accurate methods based on a one-cell approach that evaluate intracellular cytokine synthesis, our study adds a quantitative evaluation of the cytokines effectively released in the supernatant of activated $\mathrm{T}$ cells. We show high levels of IFN $\gamma$ secreted by primed T cells and, high levels of perforin. These high levels of perforin induced by fused cells suggest an activation of cytotoxic cells and an orientation of the immune response towards a cytotoxic immune response. On the other hand, we report the secretion of low levels of the inhibitory cytokine IL-10. PEG toxicity and the subsequent high levels of apoptosis, necrosis, dead cells and debris in the fusion mixture could lead to the secretion of IL-10 (31). However, recent reports suggest that IL-10 stimulates the proliferation of $\mathrm{CD}^{+} \mathrm{T}$ cells and supports a subset of $\mathrm{CD}^{+}$memory cells (32) in a synergistic way with IL-2 (33). Induction of IFN $\gamma$, IL-10 and perforin may also be explained by our choice of using total $\mathrm{CD}^{+}{ }^{+} \mathrm{T}$ cells which are necessary for a close collaboration of Th1 and Th2-type responses and for efficient anti-tumour response (34).

Therefore, maturation of DC in control wells seems to be only phenotypic and does not induce a functional maturation. Some explanations for this paradox are reviewed by Reis e Sousa who proposes to separate 'phenotypically mature' DC from 'functionally mature' ones (35). One explanation for this could be that the maturation markers could be transiently upregulated by maturing stimuli before complete maturation (36). The other explanation is that DC in control unfused wells could express substantial levels of co-stimulatory molecules and other activation markers and be tolerogenic DC (37). These hypotheses need to be further investigated in as much as maturation of DC in unfused wells may be attributable to tumour-secreted products.

In summary, the aim of this work was to characterise fusion cells generated by DC and allogeneic tumour cells. We demonstrated that imaging means are very reliable and necessary to complement flow cytometry for the characterisation of fused cells. Fused cells can be kept in vitro with a conservation of tumour and presentation antigens over time. We show that contact with tumour cells is sufficient to induce phenotypic maturation but that PEG fusion is required for effective functionality. Furthermore, we provide a quantitative dosage of cytokines and perforin secreted after $\mathrm{T}$ cell activation by fused cells. Conservation of Her $2 / n e u$ validates the use of fused cells as a breast tumour vaccine and supports the new vaccine approaches based on dendritic cells. Only fused cells are functional and appear to be good candidates for vaccination protocols however, because the number of fused cells produced was always a limiting factor, efforts must be made with the aim of improving production and standardising procedures in view of clinical trials.

\section{Acknowledgements}

K.S. was supported in part by a grant from Association pour la Recherche sur le Cancer. This work was supported by Université Paris 6 and ACTT (Amis du Centre des Tumeurs de Tenon) grants. Authors would like to thank B. Gouritin for technical assistance and S. Louis for editing assistance.

\section{References}

1. World Health Organisation Report: http://www.who.int/whr/en/ 2005.

2. Mayordomo JI, Zorina T, Storkus WJ, et al: Bone marrowderived dendritic cells pulsed with synthetic tumour peptides elicit protective and therapeutic antitumour immunity. Nat Med 1: 1297-1302, 1995 .

3. Muraro S, Bondanza A, Bellone M, Greenberg PD and Bonini C: Molecular modification of idiotypes from B-cell lymphomas for expression in mature dendritic cells as a strategy to induce tumor-reactive $\mathrm{CD}^{+}$and $\mathrm{CD} 8^{+} \mathrm{T}$-cell responses. Blood 105 : 3596-3604, 2005.

4. Paglia P, Chiodoni C, Rodolfo M and Colombo MP: Murine dendritic cells loaded in vitro with soluble protein prime cytotoxic T lymphocytes against tumor antigen in vivo. J Exp Med 183: 317-322, 1996.

5. Tuting T, Wilson CC, Martin DM, et al: Autologous human monocyte-derived dendritic cells genetically modified to express melanoma antigens elicit primary cytotoxic $\mathrm{T}$ cell responses in vitro: enhancement by cotransfection of genes encoding the Th1-biasing cytokines IL-12 and IFN-alpha. J Immunol 160: 1139-1147, 1998 .

6. Nair SK, Heiser A, Boczkowski D, et al: Induction of cytotoxic $\mathrm{T}$ cell responses and tumor immunity against unrelated tumors using telomerase reverse transcriptase RNA transfected dendritic cells. Nat Med 6: 1011-1017, 2000.

7. Holtl L, Zelle-Rieser C, Gander H, et al: Immunotherapy of metastatic renal cell carcinoma with tumor lysate-pulsed autologous dendritic cells. Clin Cancer Res 8: 3369-3376, 2002.

8. Zitvogel L, Regnault A, Lozier A, et al: Eradication of established murine tumors using a novel cell-free vaccine: dendritic cellderived exosomes. Nat Med 4: 594-600, 1998.

9. Albert ML, Sauter B and Bhardwaj N: Dendritic cells acquire antigen from apoptotic cells and induce class I-restricted CTLs. Nature 392: 86-89, 1998.

10. Gong J, Chen D, Kashiwaba M and Kufe D: Induction of antitumor activity by immunization with fusions of dendritic and carcinoma cells. Nat Med 3: 558-561, 1997.

11. Galea-Lauri J, Darling D, Mufti G, Harrison P and Farzaneh F: Eliciting cytotoxic T lymphocytes against acute myeloid leukemia-derived antigens: evaluation of dendritic cell-leukemia cell hybrids and other antigen-loading strategies for dendritic cellbased vaccination. Cancer Immunol Immunother 51: 299-310, 2002.

12. Vasir B, Borges V, Wu Z, et al: Fusion of dendritic cells with multiple myeloma cells results in maturation and enhanced antigen presentation. Br J Haematol 129: 687-700, 2005.

13. Scott-Taylor TH, Pettengell R, Clarke I, et al: Human tumour and dendritic cell hybrids generated by electrofusion: potential for cancer vaccines. Biochim Biophys Acta 1500: 265-279, 2000.

14. Shimizu K, Kuriyama H, Kjaergaard J, Lee W, Tanaka H and Shu S: Comparative analysis of antigen loading strategies of dendritic cells for tumor immunotherapy. J Immunother 27: 265-272, 2004.

15. Cheong SC, Blangenois I, Franssen JD, et al: Generation of cell hybrids via a fusogenic cell line. J Gene Med 8: 919-928, 2006.

16. Phan V, Errington F, Cheong SC, et al: A new genetic method to generate and isolate small, short-lived but highly potent dendritic cell-tumor cell hybrid vaccines. Nat Med 9: 1215-1219, 2003.

17. Koido S, Hara E, Torii A, et al: Induction of antigen-specific CD4- and CD8-mediated T-cell responses by fusions of autologous dendritic cells and metastatic colorectal cancer cells. Int J Cancer 117: 587-595, 2005.

18. Tanaka Y, Koido S, Ohana M, Liu C and Gong J: Induction of impaired antitumor immunity by fusion of MHC class II-deficient dendritic cells with tumor cells. J Immunol 174: 1274-1280, 2005 . 
19. Avigan D, Vasir B, Gong J, et al: Fusion cell vaccination of patients with metastatic breast and renal cancer induces immunological and clinical responses. Clin Cancer Res 10: 4699-4708, 2004.

20. Trefzer U, Herberth G, Wohlan K, et al: Vaccination with hybrids of tumor and dendritic cells induces tumor-specific T-cell and clinical responses in melanoma stage III and IV patients. Int J Cancer 110: 730-740, 2004.

21. Koido S, Ohana M, Liu C, et al: Dendritic cells fused with human cancer cells: morphology, antigen expression, and $\mathrm{T}$ cell stimulation. Clin Immunol 113: 261-269, 2004.

22. Raje N, Hideshima T, Davies FE, et al: Tumour cell/dendritic cell fusions as a vaccination strategy for multiple myeloma. Br J Haematol 125: 343-352, 2004

23. Tanaka H, Shimizu K, Hayashi T and Shu S: Therapeutic immune response induced by electrofusion of dendritic and tumor cells. Cell Immunol 220: 1-12, 2002.

24. Koido S, Hara E, Homma S, et al: Dendritic cells fused with allogeneic colorectal cancer cell line present multiple colorectal cancer-specific antigens and induce antitumor immunity against autologous tumor cells. Clin Cancer Res 11: 7891-7900, 2005.

25. Adams GP and Weiner LM: Monoclonal antibody therapy of cancer. Nat Biotechnol 23: 1147-1157, 2005.

26. Gong J, Avigan D, Chen D, et al: Activation of antitumor cytotoxic T lymphocytes by fusions of human dendritic cells and breast carcinoma cells. Proc Natl Acad Sci USA 97: 2715-2718, 2000.

27. Chen L, McGowan P, Ashe S, Johnston JV, Hellstrom I and Hellstrom KE: B7-1/CD80-transduced tumor cells elicit better systemic immunity than wild-type tumor cells admixed with Corynebacterium parvum. Cancer Res 54: 5420-5423, 1994.

28. Aladin F, Lautscham G, Humphries E, Coulson J and Blake N: Targeting tumour cells with defects in the MHC class I antigen processing pathway with $\mathrm{CD} 8^{+} \mathrm{T}$ cells specific for hydrophobic TAP- and Tapasin-independent peptides: the requirement for directed access into the ER. Cancer Immunol Immunother 2006.
29. Riske K, Bezlyepkina N, Lipowsky R and Dimova R: Electrofusion of model lipid membranes viewed with high temporal resolution. Biophys Rev Lett 4: 387-400, 2006

30. Matsumoto S, Saito H, Tsujitani S and Ikeguchi M: Allogeneic gastric cancer cell-dendritic cell hybrids induce tumor antigen (carcinoembryonic antigen) specific CD8(+) T cells. Cancer Immunol Immunother 55: 131-139, 2006.

31. Ronchetti A, Rovere P, Iezzi G, et al: Immunogenicity of apoptotic cells in vivo: role of antigen load, antigen-presenting cells, and cytokines. J Immunol 163: 130-136, 1999.

32. Fujii S, Shimizu K, Shimizu T and Lotze MT: Interleukin-10 promotes the maintenance of antitumor CD8(+) T-cell effector function in situ. Blood 98: 2143-2151, 2001.

33. $\mathrm{Ke} \mathrm{Y,} \mathrm{Ma} \mathrm{H}$ and Kapp JA: Antigen is required for the activation of effector activities, whereas interleukin 2 is required for the maintenance of memory in ovalbumin-specific, $\mathrm{CD} 8^{+}$cytotoxic T lymphocytes. J Exp Med 187: 49-57, 1998.

34. Tatsumi T, Kierstead LS, Ranieri E, et al: Disease-associated bias in T helper type $1(\mathrm{Th} 1) / \mathrm{Th} 2 \mathrm{CD} 4(+) \mathrm{T}$ cell responses against MAGE-6 in HLA-DRB10401(+) patients with renal cell carcinoma or melanoma. J Exp Med 196: 619-628, 2002.

35. Reis e Sousa C: Dendritic cells in a mature age. Nat Rev Immunol 6: 476-483, 2006

36. Inaba K, Turley S, Iyoda $\mathrm{T}$, et al: The formation of immunogenic major histocompatibility complex class II-peptide ligands in lysosomal compartments of dendritic cells is regulated by inflammatory stimuli. J Exp Med 191: 927-936, 2000.

37. Menges M, Rossner S, Voigtlander C, et al: Repetitive injections of dendritic cells matured with tumor necrosis factor alpha induce antigen-specific protection of mice from autoimmunity. J Exp Med 195: 15-21, 2002. 